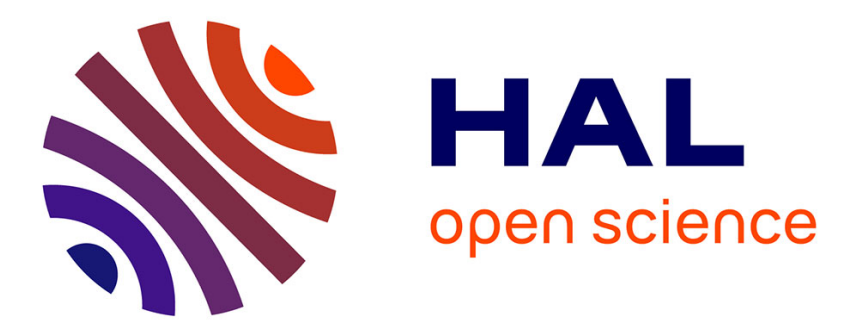

\title{
Transient microstructural brain anomalies and epileptiform discharges in mice defective for epilepsy and language-related NMDA receptor subunit gene Grin2a
}

Manal Salmi, Radu Bolbos, Sylvian Bauer, Marat Minlebaev, Nail Burnashev, Pierre Szepetowski

\section{To cite this version:}

Manal Salmi, Radu Bolbos, Sylvian Bauer, Marat Minlebaev, Nail Burnashev, et al.. Transient microstructural brain anomalies and epileptiform discharges in mice defective for epilepsy and language-related NMDA receptor subunit gene Grin2a. Epilepsia, 2018, 59 (10), pp.1919-1930. 10.1111/epi.14543 . hal-01963877

\section{HAL Id: hal-01963877 \\ https://hal-amu.archives-ouvertes.fr/hal-01963877}

Submitted on 21 Dec 2018

HAL is a multi-disciplinary open access archive for the deposit and dissemination of scientific research documents, whether they are published or not. The documents may come from teaching and research institutions in France or abroad, or from public or private research centers.
L'archive ouverte pluridisciplinaire HAL, est destinée au dépôt et à la diffusion de documents scientifiques de niveau recherche, publiés ou non, émanant des établissements d'enseignement et de recherche français ou étrangers, des laboratoires publics ou privés.

\section{(c)(1)}

Distributed under a Creative Commons Attribution| 4.0 International License 


\title{
Epilepsia
}

\section{Transient microstructural brain anomalies and epileptiform discharges in mice defective for epilepsy and language-related NMDA receptor subunit gene Grin2a}

\author{
Manal Salmi $^{1}$ | Radu Bolbos ${ }^{2}$ | Sylvian Bauer1 | Marat Minlebaev ${ }^{1,3}$ | Nail Burnashev ${ }^{1}$ \\ Pierre Szepetowski ${ }^{1}$
}

${ }^{1}$ INSERM, UMR1249, INMED, Aix-

Marseille University, Marseille, France

${ }^{2}$ CERMEP-Life Imaging Center, Lyon,

France

${ }^{3}$ Laboratory of Neurobiology, Kazan

Federal University, Kazan, Russia

Correspondence: Pierre Szepetowski, Institut de Neurobiologie de la

Méditerranée (INMED), Inserm U1249, Aix-Marseille University, Parc Scientifique de Luminy, BP13, 13273 Marseille Cedex 09, France (pierre.szepetowski@inserm.fr).

\section{Funding information}

Fédération pour la Recherche sur le Cerveau, Grant/Award Number:

R15018AA; Institut National de la Santé et de la Recherche Médicale; European Union Seventh Framework Programme FP7/2007-2013, Grant/Award Number: DESIRE/602531; Agence Nationale de la Recherche, Grant/Award Number: AMIDEX ANR-11-IDEX-0001-02

\section{Summary}

Objective: The epilepsy-aphasia spectrum (EAS) is a heterogeneous group of agedependent childhood disorders characterized by sleep-activated discharges associated with infrequent seizures and language, cognitive, and behavioral deficits. Defects in the GRIN2A gene, encoding a subunit of glutamate-gated $N$-methyl-D-aspartate (NMDA) receptors, represent the most important cause of EAS identified so far. Neocortical or thalamic lesions were detected in a subset of severe EAS disorders, and more subtle anomalies were reported in patients with so-called "benign" phenotypes. However, whether brain structural alterations exist in the context of GRIN2A defects is unknown.

Methods: Magnetic resonance diffusion tensor imaging (MR-DTI) was used to perform longitudinal analysis of the brain at 3 developmental timepoints in living mice genetically knocked out (KO) for Grin $2 a$. In addition, electroencephalography (EEG) was recorded using multisite extracellular electrodes to characterize the neocortical activity in vivo.

Results: Microstructural alterations were detected in the neocortex, the corpus callosum, the hippocampus, and the thalamus of Grin2a KO mice. Most MR-DTI alterations were detected at a specific developmental stage when mice were aged 30 days, but not at earlier (15 days) or later (2 months) ages. EEG analysis detected epileptiform discharges in Grin $2 a$ KO mice in the third postnatal week.

Significance: Grin $2 a$ KO mice replicated several anomalies found in patients with EAS disorders. Transient structural alterations detected by MR-DTI recalled the agedependent course of EAS disorders, which in humans start during childhood and show variable outcome at the onset of adolescence. Together with the epileptiform discharges detected in young Grin $2 a \mathrm{KO}$ mice, our data suggested the existence of early anomalies in the maturation of the neocortical and thalamocortical systems. Whereas the possible relationship of those anomalies with sleep warrants further investigations, our data suggest that Grin $2 a$ KO mice may serve as an animal model to study the neuronal mechanisms of EAS disorders and to design new therapeutic strategies.

\section{K E Y W O R D S}

brain structure, EEG, epilepsy-aphasia, mouse model, MR-DTI 


\section{1 | INTRODUCTION}

The epilepsy-aphasia spectrum (EAS) represents a group of heterogeneous disorders characterized by the onset during childhood, after a period of apparently normal development, of paroxysmal electroencephalographic discharges activated in sleep, associated with infrequent epileptic seizures and with speech, language, neuropsychological, and behavioral deficits that may persist in adulthood. At the more benign end of the spectrum, epilepsy with centrotemporal spikes, also known as rolandic epilepsy, is the most common focal epilepsy syndrome in childhood. Two closely related epileptic encephalopathies known as the Landau-Kleffner syndrome (LKS) and the continuous spike and wave during slow sleep syndrome (CSWSS), respectively, are situated at the more severe end of the spectrum. ${ }^{1}$ In those epileptic encephalopathies, disease evolution and response to treatment are almost entirely unpredictable and slowing of cognitive function and evolution of severe behavioral disorders might be more distressing to families than the epilepsy.

In recent years, defects and variations in the GRIN2A gene, which encodes the GluN2A subunit of glutamate-gated $N$ methyl-D-aspartate (NMDA) receptors, were identified in up to $20 \%$ of patients and families with EAS. ${ }^{2-4}$ Since then, other rare gene and genome variants have been identified in some more patients. Whereas GRIN2A represents the most important cause of EAS disorders identified so far, the underlying pathophysiologic mechanisms remain poorly understood.

A subset of EAS may occur in the context of various early brain malformations and insults, such as polymicrogyria (mostly perisylvian) or thalamic lesions. ${ }^{5}$ As a matter of fact, the role of the thalamocortical loop in the generation of sleep-related discharges has long been postulated. ${ }^{6}$ In turn, the early age of onset in EAS makes it likely that the discharges interfere with brain maturational processes. However, whether GRIN2A genetic defects are actually associated with brain structural alterations remains elusive.

Generally, developmental and pathologic processes might influence the microstructural composition and architecture of the affected brain areas. Magnetic resonance (MR) diffusion tensor imaging (DTI) is a 3-dimensional neuroimaging technique based on a Gaussian model of water molecule diffusion. DTI is noninvasive and provides information on brain microstructure and connectivity ${ }^{7-10}$ and hence can be used to characterize modifications in tissue microstructure associated with brain diseases. Besides its clinical use, DTI has also been used in basic research on brain development in rodents ${ }^{11-14}$ and to study animal models for various neurologic disorders. To look for the possible existence of brain microstructural changes associated with EAS-related genetic defects in vivo, we performed longitudinal analysis of MRI coupled with DTI of

\section{Key Points}

- Transient brain anomalies were seen in mice KO for Grin2a, the epilepsy-aphasia-related gene encoding a subunit of NMDA receptors

- Most microstructural abnormalities detected by longitudinal MR-DTI were seen in mice aged 1 month, but neither earlier nor later

- Anomalies were detected in the neocortex, the corpus callosum, the hippocampus, and the thalamus of Grin $2 a$ KO mice

- Grin2a KO mice also exhibited neocortical epileptiform discharges in the third postnatal week

the brain at different developmental timepoints in genetically engineered mice knocked out for Grin $2 a$. Because patients with GRIN2A pathogenic defects usually display epileptic discharges in childhood, electrical neocortical activity was also recorded in Grin $2 a$ KO pups.

\section{2 | MATERIAL AND METHODS}

\section{1 | Ethical statement}

Animal experimentations were performed in accordance with the French legislation and in compliance with the European Communities Council Directives (2010/63/UE). The principles outlined in the ARRIVE (Animal Research: Reporting of In Vivo Experiments) guidelines and the Basel declaration (http://www.basel.declaration.org) including the replacement, reduction and refinement (3R) concept have been considered when planning the experiments. All surgical procedures were done under anesthesia, and all efforts were made to reduce suffering. This study was approved under the French department of agriculture and the local veterinary authorities by the Animal Experimentation Ethics Committee $n^{\circ} 14$ under license $n^{\circ} 02308.03$. More detailed information on the procedure used to perform magnetic resonance diffusion tensor imaging (MR-DTI) and electroencephalography (EEG) experiments is given as supporting information Appendix S1.

\section{2 | Genotyping}

Genotyping was performed by polymerase chain reaction followed by gel electrophoresis to distinguish between the wild-type (WT), Grin $2 a^{-/-}$, and Grin $2 a^{+/-}$mice using the following combination of primers: $5^{\prime}$-tctggggcetggtcttcaaca attctgtgc, 5'-gcctgcttgccgaatatcatggtggaaaat, and 5'-cccgtt 
agcccgttgagtcacccct. All 3 cohorts were of the same C57BL/ $6 \mathrm{~N}$ genetic background, were mated and hosted in the same animal facilities, and were submitted to the same experimental procedures. There was no statistically significant difference between the 3 cohorts in sex ratio (Fisher's exact test, 2-tailed: $P=0.26)$ or in body weights at postnatal days (P) 15, P30, and P56 ( $P>0.99$ for each, 2-way analysis of variance [ANOVA] test with Tukey post hoc analysis) (Table S1).

\section{3 | EEG recordings and analyses}

Local field potential recordings were performed in headrestrained, nonanesthetized mice under analgesia, using 16site linear silicon probe placed into the somatosensory cortex to trace the columnar activity at all cortical layers. After signal amplification, filtering, and sampling, recordings were analyzed and the raw data preprocessed using the MATLAB software environment (The MathWorks, Natick, Massachusetts) followed and implemented with the experimenter's visual examination to identify and characterize epileptiform discharges. More detailed information is given as supporting information in Appendix S1.

\section{4 | MR-DTI experiments and data postprocessing}

Acquisitions were performed in vivo on a horizontal 7T BRUKER Biospec MRI system (Bruker Biospin MRI, Ettlingen, Germany) equipped with a set of gradients of $400 \mathrm{mT} / \mathrm{m}$ and controlled via Bruker ParaVision 5.1 workstation, as detailed further as supporting information in Appendix S1. Volumetric measurements of the total brains and of 4 different regions of interest (ROIs; cortex, hippocampus, corpus callosum, and thalamus) were obtained at 3 postnatal developmental stages (P15, P30, and P56) as detailed in the supporting information in Appendix S1. Values were expressed in $\mathrm{mm}^{3}$, and the relative volume of each ROI was normalized to each corresponding total volume of the brain. Diffusion tensor images were reconstructed and analyzed as described in the supporting information in Appendix S1. The 4 following DTI parameters were quantified:

Fractional anisotropy (FA) is a scalar value between 0 (isotropic) and 1 (anisotropic) that describes the degree of anisotropy of diffusion process of water molecules. FA is calculated based on the following formula, where $\lambda_{i}$ represent the 3 eigenvalues $\left(\lambda_{1}, \lambda_{2}, \lambda_{3}\right)$ of the diffusion tensor ${ }^{15}$ :

$$
\mathrm{FA}=\sqrt{\frac{1}{2}} \frac{\sqrt{\left(\lambda_{1}-\lambda_{2}\right)^{2}+\left(\lambda_{2}-\lambda_{3}\right)^{2}+\left(\lambda_{3}-\lambda_{1}\right)^{2}}}{\sqrt{\lambda_{1}^{2}+\lambda_{2}^{2}+\lambda_{3}^{2}}}
$$

Mean diffusivity (MD) takes into account the fact that the diffusion process is complex in biologic tissues and reflects several different mechanisms. ${ }^{16} \mathrm{MD}$ is an overall measure of diffusion and is the mean of the 3 main eigenvalues:

$$
\mathrm{MD}=\frac{\left(\lambda_{1}+\lambda_{2}+\lambda_{3}\right)}{3}
$$

Axial diffusivity (AD) denotes the extent of diffusion in the direction of maximal diffusivity. In the white matter, this direction is typically parallel to the orientation of the axons. It corresponds to the first eigenvalue:

$$
\mathrm{AD}=\lambda_{1}
$$

Radial diffusivity (RD) is defined as the extent of diffusion perpendicular to the direction of maximal diffusivity. This is assumed to include diffusion through the axolemma and myelin, as well as through intracellular and extracellular space perpendicular to the predominant orientation of the axons. RD is calculated from the average of the second and third eigenvalues:

$$
\mathrm{RD}=\frac{\left(\lambda_{2}+\lambda_{3}\right)}{2}
$$

These 3 last diffusion parameters (MD, AD, and RD) are expressed in $\mathrm{mm}^{2} / \mathrm{s}$.

\section{5 | Immunohistochemistry, microscopy, and image analysis}

Immunohistochemistry experiments were carried out and analyzed as reported previously ${ }^{17}$ and as further detailed in the supporting information file (Figure S2).

\section{6 | Statistics}

Data were expressed as means \pm standard error of the mean (SEM) unless otherwise stated. Two-way ANOVA test with Tukey post hoc analysis was used for MR-DTI analyses to compare between the Grin $2 a^{-/-}$or Grin $2 a^{+/-}$genotypes and the WT genotype. Bonferroni-corrected significance threshold was set at 0.0125 to take into account multiple testing of the 4 DTI parameters. Fisher's exact test was used to compare the proportions of Grin $2 a^{-1-}$ and WT mice with epileptiform discharges, and to verify the lack of differences in sex ratio between the 3 cohorts. Mann-Whitney test, 2-tailed, was used to compare the amounts of myelin basic protein (MBP) in the corpus callosum of $\operatorname{Grin} 2 a^{-1-}$ and WT mice. Kruskal-Wallis test was used to verify the lack of differences in body weights between the 3 cohorts.

\section{3 | RESULTS}

Longitudinal analysis of brain structure by MR-DTI was performed in 3 cohorts of Grin $2 a$ homozygous $\left(\right.$ Grin $2 a^{-1-}$; 
$\mathrm{n}=11)$ and heterozygous $\left(\operatorname{Grin} 2 a^{+/-} ; \mathrm{n}=7\right)$ mice and their WT $(\mathrm{n}=11)$ counterparts. Mice were subjected to neuroimaging evaluation at 2 weeks of age (P15), and then at 1 month (P30) and 8 weeks (P56) of age (Figure 1; Figure S1). Hence, each animal was subjected to neuroimaging analyses at 3 successive postnatal developmental stages. A ROI-based strategy was used, and the study focused on 4 different brain areas, which were possibly related with, and/or impacted by, GRIN2A-related disorders and dysfunction: the thalamus, the cerebral cortex, the hippocampus, and the corpus callosum. In each ROI, the 4 classical and aforementioned DTI values (fractional anisotropy [FA]; $\mathrm{MD}, \mathrm{AD}$, and RD: mean, axial, and radial diffusivities, respectively) that informed the magnitude and directionality of water movements within those areas were systematically evaluated on the right and on the left sides of the brain independently. In addition, depth EEG recordings were performed with multisite silicon probes in independent series of mice in the third postnatal week (P16-P20) to test for the presence of abnormal neocortical activities.

\section{1 | Brain MR-DTI of Grin2a KO mice does not reveal significant changes at $\mathrm{P} 15$}

MRI volumetry in the cohorts of Grin $2 a^{-/-}$and Grin $2 a^{+/-}$ mice did not reveal significant differences with the WT mice in total brain volumes (Table S2). When the relative volumes of the 4 brain areas of interest were considered, decreased volume of the right cerebral cortex $(P=0.018)$ in Grin $2 a^{+/-}$mice and of the left corpus callosum $(P=0.022)$ in $G r i n 2 a^{-1-}$ mice were detected but were not confirmed in the corresponding contralateral areas (Table S3). Analysis of DTI parameters detected an increased FA on the right side of the cerebral cortex of Grin $2 a^{+/-}$mice $(P=0.009)$ but this was not statistically confirmed on the left side. None of the other DTI parameters showed any difference in any of the 4 ROIs between either Grin $2 a^{-/-}$or Grin $2 a^{+/-}$mice, or their WT counterparts. Hence at P15, neither the lack of Grin2a nor its haploinsufficiency was associated with any significant brain neuroimaging anomaly (Figures 2-5, Figure S1, Table S4).

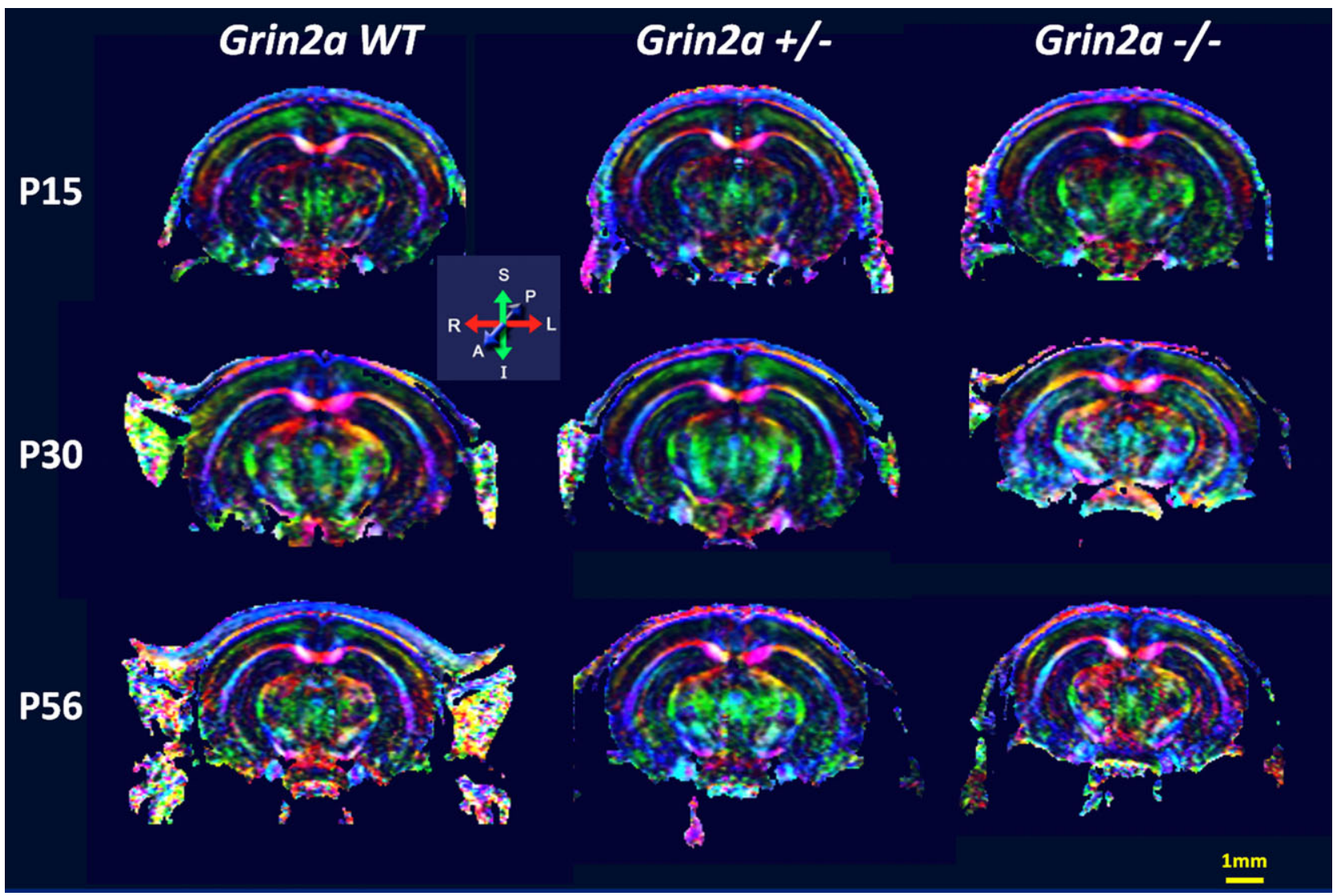

F I G URE 1 Representative color-coded fractional anisotropy maps generated from the diffusion tensor data of the brains of wild-type (WT; left column), Grin $2 a^{+/-}$(middle column) and Grin2a $a^{-/-}$(right column) mice at P15 (top line), P30, (middle line) and P56 (bottom line). The three eigenvector components are mapped as follows to the color channels: the first component (left to right or L-R) maps to red; the second component (superior to inferior or S-I) maps to green, and the third component (anterior to posterior or A-P) maps to blue. The resulting "Red, Green, Blue" (RGB) triple is assigned a transparency based on fractional anisotropy and gets finally mapped onto the map image, according to the composition rule. Color-coded maps are illustrated at $-2.45 \mathrm{~mm}$ from Bregma, as spatial coordinates based on the mouse brain in stereotaxic coordinates. ${ }^{46}$ Bar: $1 \mathrm{~mm}$ 
CEREBRAL CORTEX
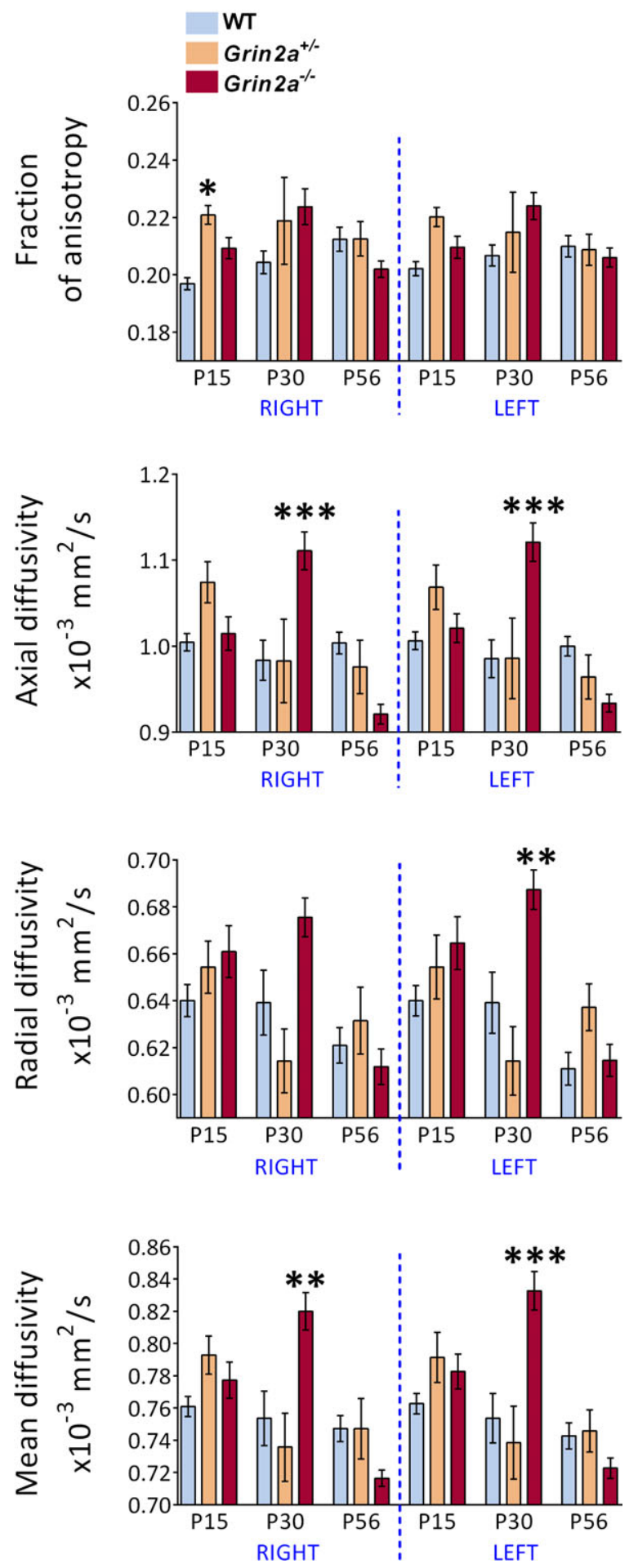

F I G URE 2 Quantitative analysis of diffusion tensor imaging (DTI) of the cerebral cortex of wild-type (WT), Grin $2 a^{+/-}$, and Grin $2 a^{-/-}$mice at P15, P30, and P56. Data are represented separately for the right and left hemispheres as mean values \pm SEM. Two-way ANOVA test with Tukey post hoc analysis followed by Bonferroni correction for multiple testing of four DTI parameters was used for comparisons between the Grin $2 a^{-1-}$ or Grin $2 a^{+/-}$genotypes and the WT genotype. $* * * P<0.00025 ; * * P<0.0025 ; * P<0.0125$

\section{CORPUS CALLOSUM}
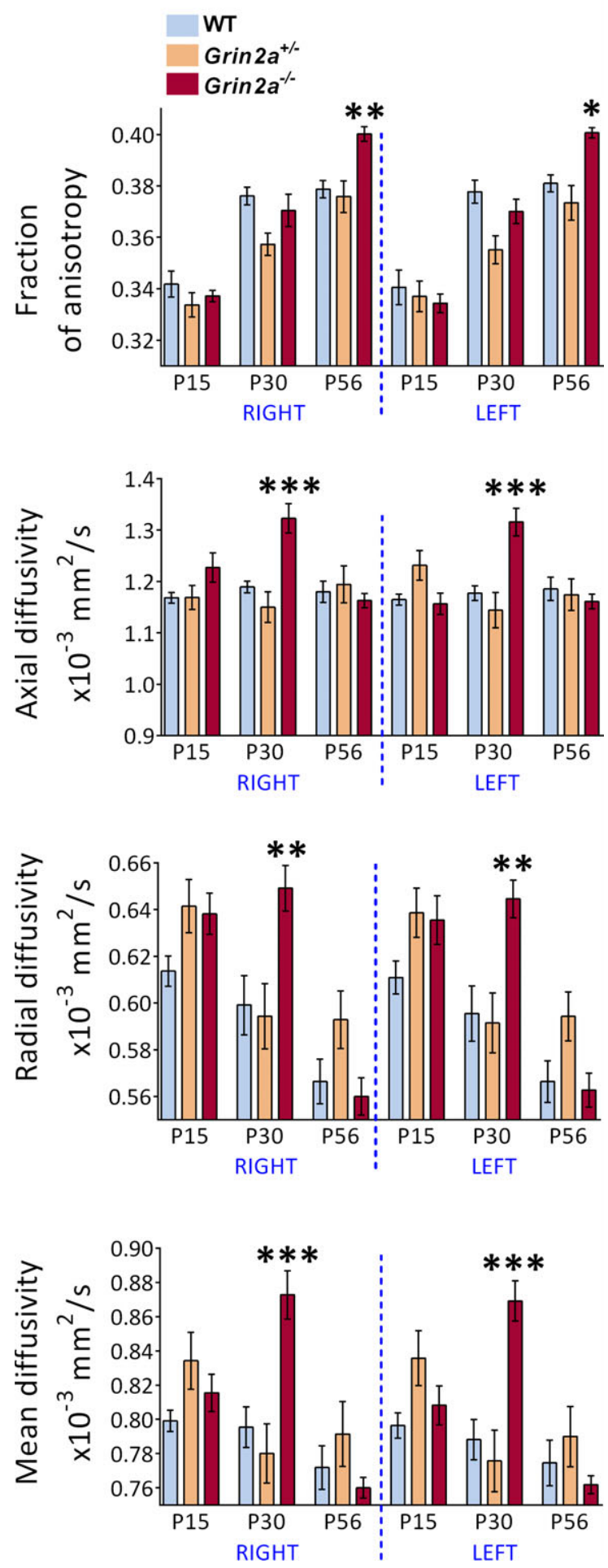

F I G URE 3 Quantitative analysis of diffusion tensor imaging (DTI) of the corpus callosum of wild-type (WT), Grin2 ${ }^{+/-}$, and Grin $2 a^{-/-}$mice at P15, P30, and P56. Data are represented separately for the right and left hemispheres as mean values \pm SEM. Two-way ANOVA test with Tukey post hoc analysis followed by Bonferroni correction for multiple testing of four DTI parameters was used for comparisons between the Grin $2 a^{-1-}$ or Grin $2 a^{+/-}$genotypes and the WT genotype. $* * * P<0.00025$; $* * P<0.0025$; $* P<0.0125$ 


\section{HIPPOCAMPUS}
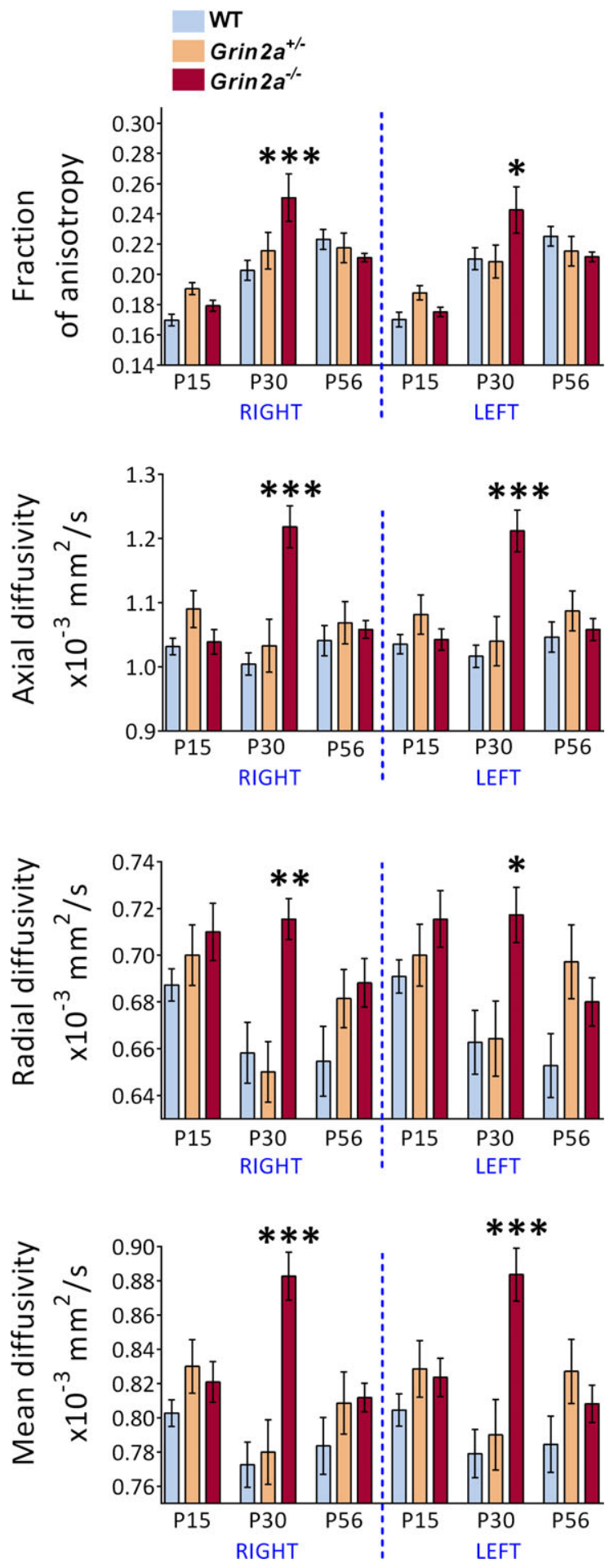

F I G URE 4 Quantitative analysis of diffusion tensor imaging (DTI) of the hippocampus of wild-type (WT), Grin2 $a^{+/-}$, and Grin2 $a^{-/-}$mice at P15, P30, and P56. Data are represented separately for the right and left hemispheres as mean values \pm SEM. Two-way ANOVA test with Tukey post hoc analysis followed by Bonferroni correction for multiple testing of 4 DTI parameters was used for comparisons between the Grin $2 a^{-/-}$or Grin $2 a^{+/-}$genotypes and the WT genotype. $* * * P<0.00025$; $* * P<0.0025$; $* P<0.0125$

\section{THALAMUS}
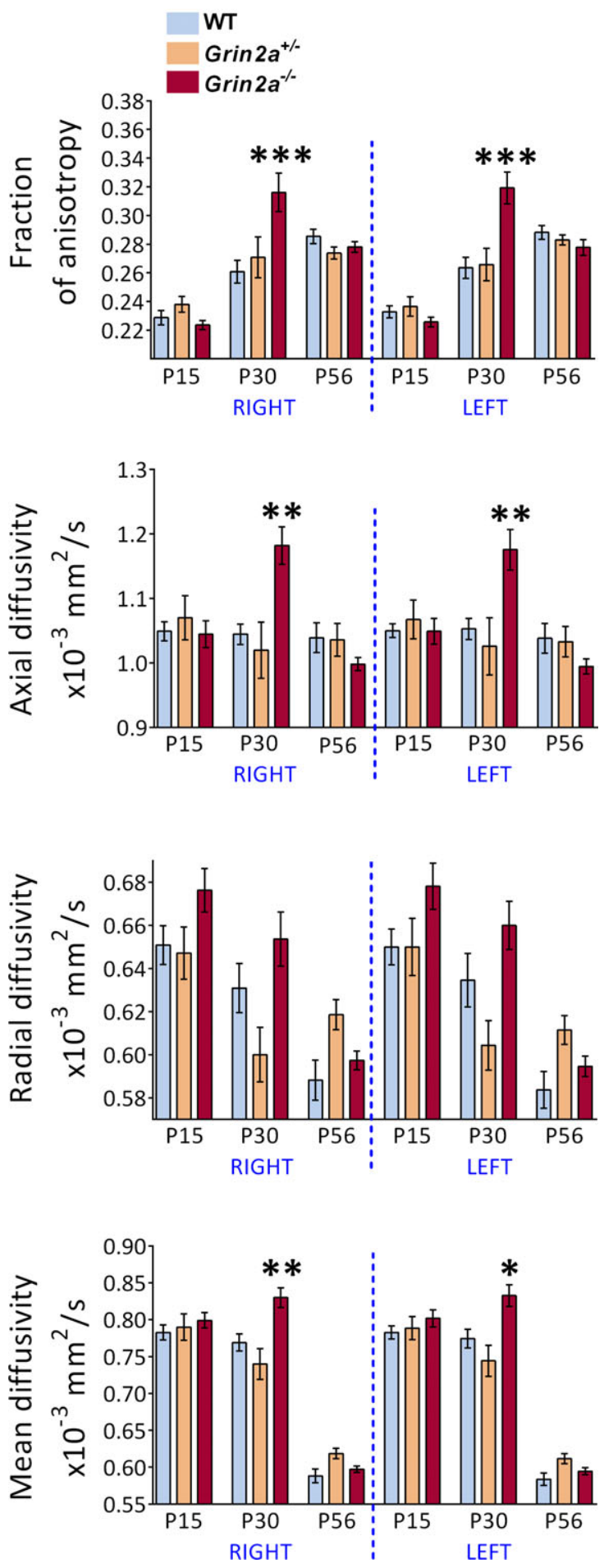

FIGURE 5 Quantitative analysis of diffusion tensor imaging (DTI) of the thalamus of wild-type (WT), Grin $2 a^{+/-}$, and Grin $2 a^{-/-}$ mice at P15, P30, and P56. Data are represented separately for the right and left hemispheres as mean values \pm SEM. Two-way ANOVA test with Tukey post hoc analysis followed by Bonferroni correction for multiple testing of 4 DTI parameters was used for comparisons between the Grin $2 a^{-1-}$ or $\operatorname{Grin} 2 a^{+/-}$genotypes and the WT genotype. $* * * P<0.00025 ; * * P<0.0025$; $* P<0.0125$ 


\section{2 | Detection of multiple MR-DTI anomalies in the cerebral cortex, the corpus callosum, the hippocampus, and the thalamus of Grin2a KO mice at P30}

Two weeks later, the same Grin $2 a^{-1-}$ mice exhibited numerous microstructural changes in all 4 ROIs as compared with the WT cohort (Figures 2-5, Figure S1, Table S4). Significant increases of the MD and of the AD were detected in the cerebral cortex on both sides simultaneously (MD: right, $P=0.0003$ and left, $P<0.0001$; AD: right, $P=0.0001$ and left, $P<0.0001$ ), whereas the concomitant increase in the FA did not resist correction for multiple testing (right, $P=0.019$ and left, $P=0.035$ ). The increased RD detected in the left cerebral cortex $(P=0.0015)$ was also seen but was not statistically confirmed after correction on the right side $(P=0.0265)$. In the corpus callosum, $\mathrm{MD}, \mathrm{AD}$, and $\mathrm{RD}$ were all increased on both sides (MD: right, $P<0.0001$ and left, $P<0.0001$; AD: right, $P<0.0001$ and left, $P<0.0001$; RD: right, $P=0.0012$ and left, $P=0.0007$ ), whereas FA was not significantly modified on both sides. In the right and left hippocampi, a significant increase in all 4 DTI parameters was detected (FA: right, $P=0.0001$ and left, $P=0.0106$; MD: right, $P<0.0001$ and left, $P<0.0001$; AD: right, $P<0.0001$ and left, $P<0.0001$; RD: right, $P=0.0015$ and left, $P=0.0048)$. Similarly, FA, MD, and AD were all increased, both in the right (FA, $P<0.0001$; MD, $P=0.0011 ; \mathrm{AD}, P=0.0011)$ and in the left thalamus (FA, $P<0.0001$; MD, $P=0.0028$; AD, $P=0.0005$ ). When volumetry was analyzed, a significant decrease in the relative volume of the left $(P=0.0015)$ and right $(P=0.016)$ thalamus was detected (Table S3). In contrast with all the aforementioned changes that were detected in the homozygous Grin $2 a^{-1-}$ mice, Grin $2 a^{+/-}$heterozygous mice did not exhibit significant DTI modifications at P30, and the decreased FA seen on the right and left corpus callosum did not resist statistical correction $(P=0.015$ and 0.0324 , respectively); however, an increase in the relative volume of the hippocampus was significantly detected in Grin $2 a^{+/-}$mice by MRI analysis on both sides of the brain (right: $P=0.0058$; left: $P=0.0009$ ), whereas the increase in the relative volume of the right corpus callosum $(P=0.034)$ was not confirmed on the left side (Figures 25, Tables S3 and S4).

\section{3 | Most MR-DTI anomalies seen at P30 in Grin2a KO mice are no longer detected 1 month later}

MR-DTI analysis was then performed on the same animals 4 weeks later (P56) (Figures 2-5, Figure S1, Table S4). In contrast with the situation encountered at
P30, all DTI values obtained in the cerebral cortex, the hippocampus, and the thalamus were no longer statistically different between either $\operatorname{Grin} 2 a^{-1-}$ or $\operatorname{Grin} 2 a^{+/-}$ mice on the one hand and WT mice on the other hand. Volumetric analysis still revealed an increased volume of the right $(P=0.013)$ but not of the left hippocampus in Grin $2 a^{+/-}$mice, whereas the decreased relative volume of the thalamus seen at P30 in Grin $2 a^{-/-}$mice was no longer detected at P56. In the corpus callosum, MD, $\mathrm{AD}$, and RD that were all increased at P30 in Grin $2 a^{-1-}$ mice, were no longer different at P56; however, Grin $2 a^{-/-}$mice displayed a significant increase of FA on both sides of the corpus callosum (right: $P=0.0012$; left: $P=0.009$ ). Immunohistochemistry experiments performed on brain samples taken from the same mice at the end of the longitudinal MR-DTI analysis did not detect significant quantitative differences in the myelin content of the corpus callosum between Grin2a $a^{-1-}$ and WT mice (Figure S2). Overall, the burst of microstructural anomalies detected in the cerebral cortex, the hippocampus, and the thalamus of $\mathrm{Grin} 2 a^{-/-}$mice aged 1 month had spontaneously and completely remitted 1 month later, whereas the corpus callosum still showed DTI anomalies, albeit different from those detected at P30.

\section{4 | Grin2a KO mice exhibit epileptiform discharges}

Children with EAS disorders, including those bearing GRIN2A pathogenic defects, exhibit abnormal electroencephalographic discharges that are not necessarily associated with clinical seizures. To the best of our knowledge, no epilepsy has ever been reported in Grin $2 a \mathrm{KO}$ mice. We reasoned that subclinical events might yet exist in those mice, particularly at the early developmental stages when the immature brain is more excitable and prone to discharges. ${ }^{18}$ Hence, we decided to perform neocortical EEG recordings in vivo during the third postnatal week (postnatal days P16-P20) using a 16-channel linear silicon probe (Figure 6A). The third postnatal week was investigated notably because it roughly corresponds to 2-3 years of age in humans, ${ }^{19}$ when seizures may start in patients with EAS. During the 6 hour recordings of head-restrained, nonanesthetized mice, no abnormal event was ever detected in the WT mice $(n=9)$. In contrast, epileptiform discharges were detected at least once in 5 of $11 \mathrm{Grin} 2 a^{-/-}$ mice ( $P=0.0379$, Fisher's exact test, 2-tailed) (Figure 6B). The discharge recurred once more in 2 of those 5 mice. Those rare epileptiform events had a mean duration of $7.88 \pm 2.21$ seconds and a mean amplitude of $186.06 \pm$ $11.56 \mu \mathrm{V}$ and consisted of mixed polyspike-and-waves and sharp waves with 2 major peaks of power spectrum in the $\delta-\theta$ frequency range $(4.43 \pm 0.1 \mathrm{~Hz})$ and in the $\alpha$ 


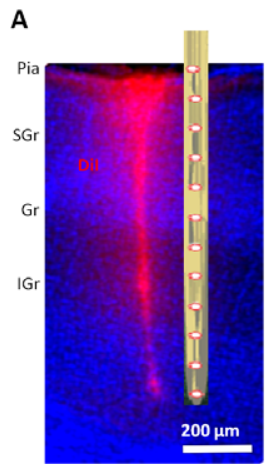

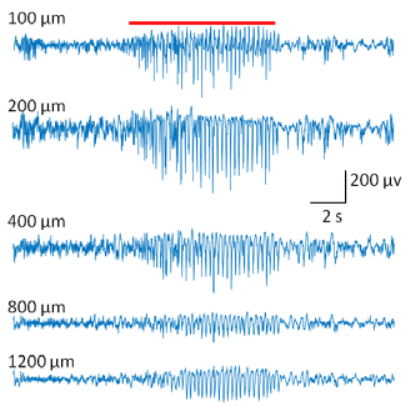

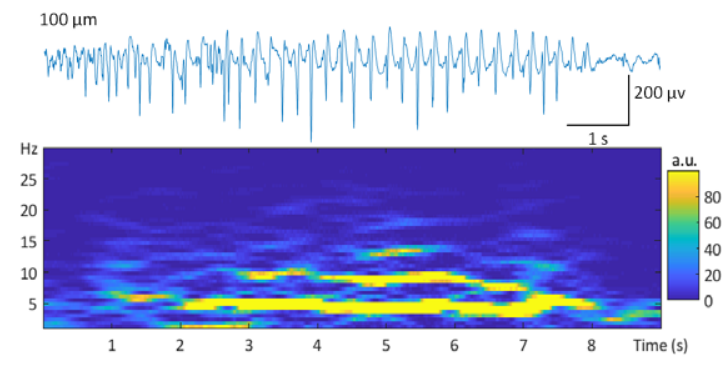

D

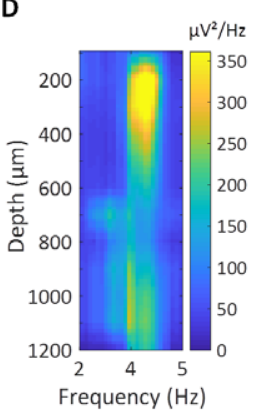

G

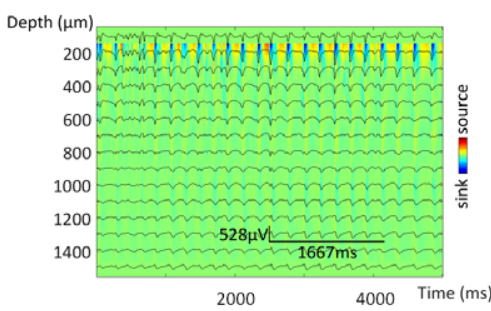

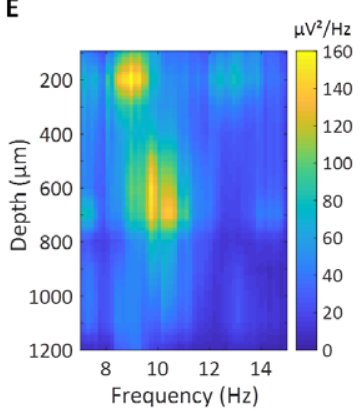

H

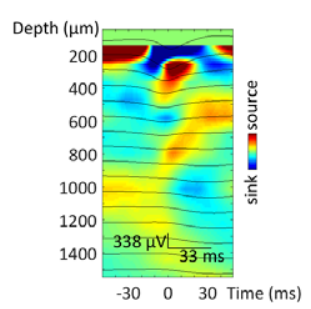

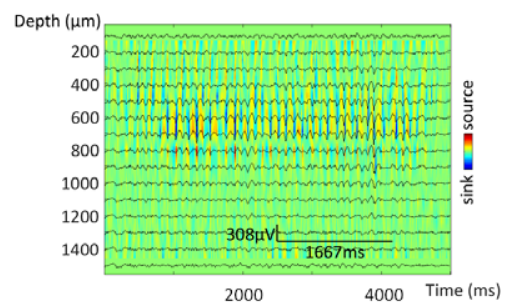

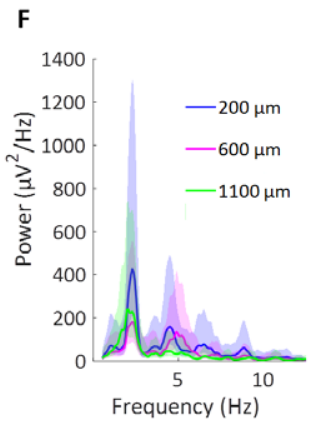

J

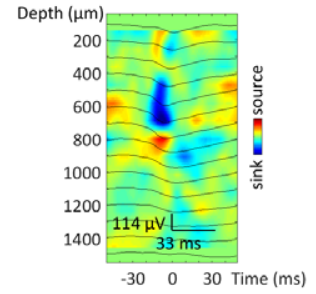

F I G U RE 6 Spontaneous epileptiform discharges in Grin $2 a^{-1-}$ mice. Local field potential (LFP) recordings were performed from the somatosensory cortices of head-restrained Grin $2 a^{-/-}$mice aged P16 to P20. Epileptiform discharges were detected in 5 of 11 Grin2 $a^{-/-}$mice at different neocortical depths. No epileptiform discharge was detected in WT mice $(\mathrm{n}=9)(P=0.0379$, Fisher's exact test, 2-tailed). A, Experimental setup using 16-channel silicon probe recordings from the somatosensory cortex in a Grin $2 a^{-1-}$ mouse. The upper channel corresponds to the superficial intracortical electrode placed at $100 \mu \mathrm{m}$ from the pia. Hoechst staining was used to distinguish between the supragranular (SGr), granular (Gr), and infragranular (IGr) neocortical layers. DiI staining was used to visualize the position of the silicon probe. B, Epileptiform discharge (red line) recorded in a Grin $2 a^{-1-}$ mouse at the depths as indicated on the left side of each corresponding trace. C, Time-frequency spectrogram of the epileptiform discharge shown in (B) at depth $100 \mu \mathrm{m}$ from pia. a.u., arbitrary units. D,E, Color-coded maps of the mean power spectrum of the epileptiform discharges detected in Grin $2 a^{-1-}$ mice throughout the neocortical depth. D, Contribution of the $\delta-\theta$ frequency band $(2-6 \mathrm{~Hz})$ in power spectrum during discharges was stronger in the supragranular and infragranular neocortical layers. E, Contribution of the $\alpha$ frequency band $(8-15 \mathrm{~Hz})$ was stronger in the supragranular and granular neocortical layers. F, Distribution of mean power spectral density of the epileptiform discharges detected in Grin $2 a^{-1-}$ mice showed 2 major peaks in the $\delta-\theta(4.43 \pm 0.1 \mathrm{~Hz})$ and $\alpha$ $(10.37 \pm 0.14 \mathrm{~Hz})$ frequency bands, at depths corresponding to the supragranular $(200 \mu \mathrm{m})$, granular $(600 \mu \mathrm{m})$, and infragranular $(1100 \mu \mathrm{m})$

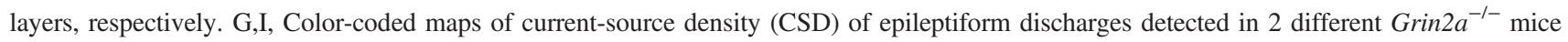
throughout the neocortical depth. H,J, Averaged population spikes at different cortical depths (black traces) superimposed with the corresponding CSD maps of the epileptiform discharges shown in (G) and (I), respectively

frequency range $(10.37 \pm 0.14 \mathrm{~Hz}$ ), respectively (Figure $6 \mathrm{C}-\mathrm{F}$ ). Contribution of the $\delta-\theta$ band in power spectrum during discharges was stronger in the supragranular and infragranular neocortical layers (Figure 6D,F), whereas contribution of the $\alpha$ band was stronger in the supragranular and granular neocortical layers (Figure 6E,F). Consistently, current source density analyses predominantly detected sinks at depths roughly corresponding to the depths of maximal power of the detected epileptiform events (Figure 6G-J). 


\section{4 | DISCUSSION}

GRIN2A genetic defects cause EAS disorders in humans. In this study, we have used longitudinal MR-DTI analysis and depth EEG recordings in vivo to show that the $\mathrm{KO}$ of the murine Grin2a gene, encoding the GluN2A subunit of NMDA receptors, is associated with microstructural and functional alterations in brain areas of interest with respect to the corresponding human pathology. Longitudinal imaging studies are particularly useful in the context of developmental and pathologic age-dependent processes but are usually expensive and not easy to perform in human patients. Likewise, local field potential recordings to trace the columnar activity at all cortical layers are difficult or even impossible in human patients. Relevant animal models such as the one studied here can thus be helpful to study the structural and functional changes that occur in the course of EAS disorders.

Interpretation of the DTI changes that were observed is inherently difficult. DTI analysis shows high sensitivity but low specificity. Whereas reduced FA has been frequently detected in a broad range of brain disorders, increases in FA as seen here have been reported more rarely. In the thalamus particularly, which is a fiber-rich structure, increased FA was observed in paroxysmal kinesigenic dyskinesia, ${ }^{20}$ presymptomatic Alzheimer's disease, ${ }^{21}$ childhood absence epilepsy, ${ }^{22}$ or photosensitive juvenile myoclonic epilepsy ${ }^{23}$; this pattern of increased FA could be due to a selective degeneration of white matter fibers, whereas the decreased volume of the thalamus and the accompanying increased mean diffusivity seen at $\mathrm{P} 30$ are compatible with decreased cell number in the thalamocortical system. FA changes seen at P56 in the corpus callosum of Grin2a KO mice were apparently not due to increased myelination of white fibers, although we cannot exclude that subtle changes in axon myelination might have escaped detection from immunohistochemistry experiments, especially given that the relative FA increased by about $5 \%$ on average in Grin2a KO mice at P56 (Table S4). Moreover, FA depends only partly on axon myelination, ${ }^{24}$ and other processes such as cytotoxic edema, ${ }^{25}$ organization of reactive astrocytes, ${ }^{26}$ or changes in axonal membranes ${ }^{27}$ might play more major or additive roles in the modification of anisotropic diffusion. Whereas decreased axonal density would cause the increase in axial diffusivity seen at P30 in all 4 regions of interest, the concomitant increases of several DTI parameters seen at P30 were likely caused by multiple and intertwined events such as altered myelination, axonal or cell loss, gliosis, and inflammation. Not exclusively and as fractional anisotropy and diffusivity follow age-dependent trajectories, ${ }^{28}$ as also seen in our WT mice, the transient modifications seen in the Grin $2 a$ KO mice might reflect altered maturation of the brain regions studied here.
It has indeed long been postulated that EAS disorders result from an underlying abnormality of brain maturation. It should also be mentioned that for each neuroimaging parameter, only those changes that were found different between WT and KO mice with statistical significance on both the right and the left sides of a given ROI were considered. By using this very stringent procedure, some data of biologic significance might have been excluded; indeed, asymmetrical functioning of murine brain areas has already been demonstrated, including long-term potentiation in the hippocampal CA1 area of Grin $2 a \mathrm{KO}$ mice. ${ }^{29}$ Whereas dramatic brain malformations including neocortical and thalamic abnormalities have been detected by neuroimaging in patients with so-called "symptomatic" CSWSS, no macrostructural anomaly was usually detected by MRI in patients carrying GRIN2A mutations. ${ }^{2,4,30}$ However, more subtle and transient anomalies might exist, as suggested by the present study. Indeed, microstructural neuroimaging anomalies of white matter were found by DTI in patients with RE-hence at the benign end of EAS. ${ }^{31}$ Although GRIN2A pathogenic variants are mostly found in the more severe LKS and CSWSS patients, ${ }^{4,32}$ their existence in some of the RE patients with DTI anomalies mentioned just above cannot be excluded. Of interest also, the decrease in the relative volume of the thalamus seen here at P30 recalls the smaller thalamic volumes recently reported in human patients with polymicrogyria and continuous spike and waves during sleep. ${ }^{5}$

It is important to note that most MR-DTI alterations detected here were only seen at a specific developmental time window when mice were aged 1 month (P30). Disorders of the EAS that can be caused by defects of the human GRIN2A gene include a range of clinical manifestations that are also age-limited. It is tempting to speculate that the transient burst of MR-DTI anomalies detected here in Grin2a KO mice at P30, somehow parallels the evolving presentation of EAS disorders in human. Precise temporal comparison of brain development and maturation between rodents and human has always been challenging and somehow controversial; indeed, different conclusions might be drawn depending on which developmental criteria are used, such as neuroanatomical and electrophysiologic changes, cell proliferation and migration, synaptogenesis, myelination, or age-dependent behaviors. This being kept in mind, the 4- to 11-year-old period in humans broadly corresponds to P25-P35 in rodents, ${ }^{19}$ and, based on the developmental evolution of MR-DTI parameters in C57B1/6 mice, adolescence might last until 3 months of age in such mice. ${ }^{28}$ Hence, the developmental stage (P30) when most MR-DTI anomalies were seen here in Grin2a KO mice recalls the time period of EAS disorders, with an onset between 2 and 12 years old. ${ }^{1}$ Of interest, GluN2A expression is dynamically regulated during brain development and increases 
dramatically during the second postnatal week in mice: This may well be linked with the age-dependence characteristics of the anomalies seen here and in the patients. Grin2a genetic defect could sustain neocortical hyperexcitability and a delayed maturation of the brain. Not exclusively, it was previously shown that DTI parameters might change over time as a consequence of epileptic activity ${ }^{33}$; in patients with RE, abnormal trajectory of brain structural development has indeed been reported in neocortical and subcortical areas over 2 years after onset of disease. ${ }^{34}$ As a matter of fact, increased diffusivity as detected in Grin $2 a$ $\mathrm{KO}$ mice might reflect altered maturation but is also seen classically during interictal periods in patients with focal epilepsy. ${ }^{35}$ Whereas no overt epileptic seizure has ever been reported in this Grin $2 a \mathrm{KO}$ model, our data indicated that those mice displayed epileptiform discharges in the third postnatal week, hence before the structural anomalies were detected.

Conversely, the transient modifications in the microstructure of the brain areas are likely to impact on their maturation and functioning and to influence the presentation of the epileptic, cognitive, and behavioral Grin $2 a$-related phenotypes. As an example, the transient thalamic anomalies might impact on the maturation of the thalamocortical circuitry and of slow-wave sleep notably. Of interest, experience-dependent maturation of slow-wave sleep in the mouse was shown to be Grin2a-dependent during a late critical period starting at $\mathrm{P} 30,{ }^{36}$ when the transient anomalies were detected in the present study. Thalamic anomalies might also favor the generation of epileptic activity. Hence, thalamic DTI anomalies have already been reported in absence seizures ${ }^{22,37}$ and in temporal lobe epilepsy. ${ }^{38,39}$ Functional thalamic abnormalities were also found in patients with CSWSS. ${ }^{40}$ Similarly, changes in the hippocampus and in the corpus callosum might have long-term influence on cognitive abilities; as a matter of fact, adult Grin2a KO mice show impaired spatial memory. ${ }^{41}$ In patients with Rolandic epilepsy, DTI anomalies of the white matter correlated with duration of epilepsy and with cognitive disturbances. ${ }^{31}$

Power spectral analysis of local field potential revealed increased contribution of oscillations in the delta/theta and spindle frequency ranges. Of interest, both delta and spindling activities are elements of non-REM (rapid eye movement) sleep. In children with EAS, the activation of interictal epileptiform discharges in sleep has been associated with the occurrence of spindles, ${ }^{42}$ and it was proposed that the thalamocortical circuitry that generates spindles might be hijacked to generate spike and wave discharges. ${ }^{43}$ Consistently, power spectrum and current source density analyses indicated the possible involvement of the granular neocortical layer that receives afferent connections from the thalamus. In addition, our data indicated the participation of supragranular and infragranular layers, hence suggesting an alternative and nonexclusive intracortical mechanism where epileptiform activity would propagate through horizontal pathways, as proposed previously. ${ }^{4,45}$ Whatever their mechanism(s), discharges were rarely detected-and not in all KO pups. In patients with EAS disorders, discharges occur mostly during slow-wave sleep; ongoing analyses will determine if the increased and abnormal neocortical activity seen in Grin $2 a \mathrm{KO}$ mice would be related with selective stages of the sleep-wake cycle, and with slow-wave sleep notably, and whether it would persist in later stages.

Regardless of their underlying origin, microstructural anomalies generated in the context of GRIN2A defects could represent possible biomarkers to predict the severity, the comorbidity, and the outcome of EAS disorders. Generally, our data indicate that the Grin $2 a \mathrm{KO}$ mice displayed epileptiform discharges and age-dependent structural anomalies, and might be useful as an animal model to study the pathophysiology of EAS disorders and ultimately to design new therapeutic tools.

\section{ACKNOWLEDGMENTS}

We thank S Corby and her staff at INMED animal core facilities, and JB Langlois at CERMEP. This work was supported by INSERM (Institut National de la Santé et de la Recherche Médicale), by FRC (Fédération pour la Recherche sur le Cerveau) grant (no. R15018AA), and by the European Union Seventh Framework Programme FP7/ 2007-2013 under the project DESIRE (grant agreement $\left.\mathrm{n}^{\circ} 602531\right)$. This work was carried out within the FHU EPINEXT thanks to the support of the AMIDEX project (ANR-11-IDEX-0001-02) funded by the "Investissements d'Avenir" French government program managed by the French National Research Agency (ANR). MS has been a recipient of an LFCE (Ligue Française Contre l'Epilepsie) $\mathrm{PhD}$ fellowship.

\section{DISCLOSURE}

None of the authors has any conflict of interest to disclose. We confirm that we have read the Journal's position on issues involved in ethical publication and affirm that this report is consistent with those guidelines.

\section{AUTHOR CONTRIBUTIONS}

MS and RB performed and directed experiments and analyzed the data with equal contributions. SB performed and analyzed immunohistochemistry experiments. $\mathrm{MM}$ and $\mathrm{NB}$ participated in the design and in the analysis of EEG recordings. PS was the project leader. He decided on the 
overall strategy, directed the follow-up of experiments, supervised data analysis, and wrote the manuscript with MS and RB. All authors contributed to the final version of the manuscript.

\section{OR CID}

\section{Pierre Szepetowski (iD http://orcid.org/0000-0003-2060- 8390}

\section{REFERENCES}

1. Tassinari CA, Cantalupo G, Dalla Bernardina B, et al Encephalopathy related to status epilepticus during slow sleep (ESES) including Landau-Kleffner syndrome. In: Epileptic Syndromes in Infancy, Childhood and Adolescence. Montrouge, France: John Libbey Eurotext Ltd, 2012; p. 255-75.

2. Carvill GL, Regan BM, Yendle SC, et al. GRIN2A mutations cause epilepsy-aphasia spectrum disorders. Nat Genet. 2013;45:1073-6.

3. Lemke JR, Lal D, Reinthaler EM, et al. Mutations in GRIN2A cause idiopathic focal epilepsy with rolandic spikes. Nat Genet. 2013;45:1067-72.

4. Lesca G, Rudolf G, Bruneau N, et al. GRIN2A mutations in acquired epileptic aphasia and related childhood focal epilepsies and encephalopathies with speech and language dysfunction. Nat Genet. 2013;45:1061-6.

5. Bartolini E, Falchi M, Zellini F, et al. The syndrome of polymicrogyria, thalamic hypoplasia, and epilepsy with CSWS. Neurology. 2016;86:1250-9.

6. Nieuwenhuis L, Nicolai J. The pathophysiological mechanisms of cognitive and behavioral disturbances in children with LandauKleffner syndrome or epilepsy with continuous spike-and-waves during slow-wave sleep. Seizure. 2006;15:249-58.

7. Egger K, Janz P, Dobrossy MD, et al. Microstructural effects of a neuro-modulating drug evaluated by diffusion tensor imaging. NeuroImage. 2016;127:1-10.

8. Gupta M, Mishra SK, Kumar BS, et al. Early detection of whole body radiation induced microstructural and neuroinflammatory changes in hippocampus: a diffusion tensor imaging and gene expression study. J Neurosci Res. 2017;95:1067-78.

9. Mori S, Zhang J. Principles of diffusion tensor imaging and its applications to basic neuroscience research. Neuron. 2006;51:527-39.

10. Muller HP, Vernikouskaya I, Ludolph AC, et al. Fast diffusion tensor magnetic resonance imaging of the mouse brain at ultrahigh-field: aiming at cohort studies. PLoS One. 2012;7:e53389.

11. Bockhorst KH, Narayana PA, Liu R, et al. Early postnatal development of rat brain: in vivo diffusion tensor imaging. J Neurosci Res. 2008;86:1520-8.

12. Chahboune H, Ment LR, Stewart WB, et al. Neurodevelopment of C57B/L6 mouse brain assessed by in vivo diffusion tensor imaging. NMR Biomed. 2007;20:375-82.

13. Mori S, Itoh R, Zhang J, et al. Diffusion tensor imaging of the developing mouse brain. Magn Reson Med. 2001;46:18-23.

14. Zhang J, Richards LJ, Yarowsky P, et al. Three-dimensional anatomical characterization of the developing mouse brain by diffusion tensor microimaging. NeuroImage. 2003;20:1639-48.
15. Basser PJ, Pierpaoli C. Microstructural and physiological features of tissues elucidated by quantitative-diffusion-tensor MRI. J Magn Reson. 1996;111:209-19.

16. Le Bihan D, Breton E, Lallemand D, et al. MR imaging of intravoxel incoherent motions: application to diffusion and perfusion in neurologic disorders. Radiology. 1986;161:401-7.

17. Salmi M, Bruneau N, Cillario J, et al. Tubacin prevents neuronal migration defects and epileptic activity caused by rat Srpx2 silencing in utero. Brain. 2013;136:2457-73.

18. Dulac O, Milh M, Holmes GL. Brain maturation and epilepsy. Handb Clin Neurol. 2013;111:441-6.

19. Semple BD, Blomgren K, Gimlin K, et al. Brain development in rodents and humans: identifying benchmarks of maturation and vulnerability to injury across species. Prog Neurobiol. 2013;106107:1-16.

20. Kim JH, Kim DW, Kim JB, et al. Thalamic involvement in paroxysmal kinesigenic dyskinesia: a combined structural and diffusion tensor MRI analysis. Hum Brain Mapp. 2015;36:1429-41.

21. Ryan NS, Keihaninejad S, Shakespeare TJ, et al. Magnetic resonance imaging evidence for presymptomatic change in thalamus and caudate in familial Alzheimer's disease. Brain. 2013;136:1399-414.

22. Yang T, Guo Z, Luo C, et al. White matter impairment in the basal ganglia-thalamocortical circuit of drug-naive childhood absence epilepsy. Epilepsy Res. 2012;99:267-73.

23. von Podewils F, Runge U, Kruger S, et al. Diffusion tensor imaging abnormalities in photosensitive juvenile myoclonic epilepsy. Eur J Neurol. 2015;22:1192-200.

24. Aung WY, Mar S, Benzinger TL. Diffusion tensor MRI as a biomarker in axonal and myelin damage. Imaging Med. 2013;5:427-40.

25. Mayer AR, Ling J, Mannell MV, et al. A prospective diffusion tensor imaging study in mild traumatic brain injury. Neurology. 2010;74:643-50.

26. Budde MD, Janes L, Gold E, et al. The contribution of gliosis to diffusion tensor anisotropy and tractography following traumatic brain injury: validation in the rat using Fourier analysis of stained tissue sections. Brain. 2011;134:2248-60.

27. Beaulieu $\mathrm{C}$. The basis of anisotropic water diffusion in the nervous system - a technical review. NMR Biomed. 2002;15:435-55.

28. Hammelrath L, Skokic S, Khmelinskii A, et al. Morphological maturation of the mouse brain: an in vivo MRI and histology investigation. NeuroImage. 2016;125:144-52.

29. Wu Y, Kawakami R, Shinohara Y, et al. Target-cell-specific leftright asymmetry of NMDA receptor content in schaffer collateral synapses in epsilon1/NR2A knock-out mice. J Neurosci. 2005;25:9213-26.

30. Endele S, Rosenberger G, Geider K, et al. Mutations in GRIN2A and GRIN2B encoding regulatory subunits of NMDA receptors cause variable neurodevelopmental phenotypes. Nat Genet. 2010;42:1021-6.

31. Ciumas C, Saignavongs M, Ilski F, et al. White matter development in children with benign childhood epilepsy with centro-temporal spikes. Brain. 2014;137:1095-106.

32. Burnashev N, Szepetowski P. NMDA receptor subunit mutations in neurodevelopmental disorders. Curr Opin Pharmacol. 2015;20:73-82.

33. Yu JT, Tan L. Diffusion-weighted magnetic resonance imaging demonstrates parenchymal pathophysiological changes in epilepsy. Brain Res Rev. 2008;59:34-41. 
34. Garcia-Ramos C, Jackson DC, Lin JJ, et al. Cognition and brain development in children with benign epilepsy with centrotemporal spikes. Epilepsia. 2015;56:1615-22.

35. Thivard L, Adam C, Hasboun D, et al. Interictal diffusion MRI in partial epilepsies explored with intracerebral electrodes. Brain. 2006;129:375-85.

36. Miyamoto H, Katagiri H, Hensch T. Experience-dependent slowwave sleep development. Nat Neurosci. 2003;6:553-4.

37. Luo C, Xia Y, Li Q, et al. Diffusion and volumetry abnormalities in subcortical nuclei of patients with absence seizures. Epilepsia. 2011;52:1092-9.

38. Gong G, Concha L, Beaulieu C, et al. Thalamic diffusion and volumetry in temporal lobe epilepsy with and without mesial temporal sclerosis. Epilepsy Res. 2008;80:184-93.

39. Kimiwada T, Juhasz C, Makki M, et al. Hippocampal and thalamic diffusion abnormalities in children with temporal lobe epilepsy. Epilepsia. 2006;47:167-75.

40. Agarwal R, Kumar A, Tiwari VN, et al. Thalamic abnormalities in children with continuous spike-wave during slow-wave sleep: an F-18-fluorodeoxyglucose positron emission tomography perspective. Epilepsia. 2016;57:263-71.

41. Sakimura K, Kutsuwada T, Ito I, et al. Reduced hippocampal LTP and spatial learning in mice lacking NMDA receptor epsilon 1 subunit. Nature. 1995;373:151-5.

42. Nobili L, Baglietto MG, Beelke M, et al. Spindles-inducing mechanism modulates sleep activation of interictal epileptiform discharges in the Landau-Kleffner syndrome. Epilepsia. 2000;41:201-6.
43. Beenhakker MP, Huguenard JR. Neurons that fire together also conspire together: is normal sleep circuitry hijacked to generate epilepsy? Neuron. 2009;62:612-32.

44. Borbely S, Halasy K, Somogyvari Z, et al. Laminar analysis of initiation and spread of epileptiform discharges in three in vitro models. Brain Res Bull. 2006;69:161-7.

45. Wenzel M, Hamm JP, Peterka DS, et al. Reliable and elastic propagation of cortical seizures in vivo. Cell Rep. 2017;19:2681-93.

46. Paxinos G, Franklin KBJ. Paxinos and Franklin's the Mouse Brain in Stereotaxic Coordinates. Amsterdam: Academic Press; 2013.

\section{SUPPORTING INFORMATION}

Additional supporting information may be found online in the Supporting Information section at the end of the article.

How to cite this article: Salmi M, Bolbos R, Bauer S, Minlebaev M, Burnashev N, Szepetowski $\mathrm{P}$. Transient microstructural brain anomalies and epileptiform discharges in mice defective for epilepsy and language-related NMDA receptor subunit gene Grin2a. Epilepsia. 2018;00:1-12. https://doi.org/ $\underline{10.1111 / \text { epi.14543 }}$ 